\title{
Signatures of Correct Computation
}

\author{
Charalampos Papamanthou ${ }^{1}$, Elaine $\mathrm{Shi}^{2}$, and Roberto Tamassia ${ }^{3}$ \\ 1 UC Berkeley \\ cpap@cs.berkeley. edu \\ ${ }^{2}$ University of Maryland \\ elaine@cs.umd.edu \\ 3 Brown University \\ rtacs. brown. edu
}

\begin{abstract}
We introduce Signatures of Correct Computation (SCC), a new model for verifying dynamic computations in cloud settings. In the SCC model, a trusted source outsources a function $f$ to an untrusted server, along with a public key for that function (to be used during verification). The server can then produce a succinct signature $\sigma$ vouching for the correctness of the computation of $f$, i.e., that some result $v$ is indeed the correct outcome of the function $f$ evaluated on some point $\mathbf{a}$. There are two crucial performance properties that we want to guarantee in an SCC construction: (1) verifying the signature should take asymptotically less time than evaluating the function $f$; and (2) the public key should be efficiently updated whenever the function changes.

We construct SCC schemes (satisfying the above two properties) supporting expressive manipulations over multivariate polynomials, such as polynomial evaluation and differentiation. Our constructions are adaptively secure in the random oracle model and achieve optimal updates, i.e., the function's public key can be updated in time proportional to the number of updated coefficients, without performing a linear-time computation (in the size of the polynomial).

We also show that signatures of correct computation imply Publicly Verifiable Computation (PVC), a model recently introduced in several concurrent and independent works. Roughly speaking, in the SCC model, any client can verify the signature $\sigma$ and be convinced of some computation result, whereas in the PVC model only the client that issued a query (or anyone who trusts this client) can verify that the server returned a valid signature (proof) for the answer to the query. Our techniques can be readily adapted to construct PVC schemes with adaptive security, efficient updates and without the random oracle model.
\end{abstract}

\section{Introduction}

Given the emergence of the cloud computing paradigm in business and consumer applications, it has become increasingly important to provide integrity guarantees in third-party data management settings. Consider for example the following scenario: A company has developed some novel algorithm, e.g., for personalized medicine, or for stock trend prediction. To avoid investing in expensive IT infrastructure in-house, the company chooses to outsource the execution of this algorithm to an external, untrusted cloud provider (e.g., Amazon, Google). How could a user verify the correctness of the computation under the assumption that she only trusts the company that developed the

A. Sahai (Ed.): TCC 2013, LNCS 7785, pp. 222-242, 2013.

(c) International Association for Cryptologic Research 2013 
algorithm, but not the cloud provider? The above question poses two crucial requirements: (1) efficiency, meaning that the running time of the verification algorithms executed by the client should be asymptotically less than the time needed to execute the algorithm in the cloud; and (2) public verifiability, meaning that our verification mechanism should not be tied to a specific verifier's secret key so that any user can verify the computation. In addition, another desirable property is to efficiently handle updates to the outsourced algorithm, without computing public parameters from scratch.

In this paper, we propose a new paradigm for verifying dynamic computation in the cloud called signatures of correct computation (SCC). SCC allows an untrusted worker to produce a signature vouching for the correctness of some computation over some input; any user can verify the signature using a public key (produced by an one-time preprocessing) published by a trusted source who outsourced the function in the cloud.

Signatures of correct computation are closely related to publicly verifiable computation (PVC), proposed by Parno et al. [31], Canetti et al. [9] and Fiore and Gennaro [12 13], in concurrent and independent works to ours. Specifically, signatures of correct computation are stronger than publicly verifiable computation: given an SCC scheme, one can directly construct a PVC scheme; while the other way around does not seem to be true. More specifically, in PVC, a "proof of correct computation" is tied to a specific challenge (generated by an algorithm ProbGen in [31]), and can only be verified by the client who has generated that challenge (or anyone who trusts this client). By contrast, a signature of correct computation is not tied to any challenge, and can be verified by anyone in the world, in much the same way as a traditional signature on a message. We provide a detailed comparison of PVC and SCC in Section 1.2

\subsection{Results and Contributions}

We design SCC schemes for multivariate polynomial manipulations, including polynomial evaluation and differentiation. One of our technical highlights is a new method in this setting that allows us to slightly modify our selectively secure schemes to achieve adaptive security. Our SCC schemes achieve adaptive security under the random oracle model. We also show that under the weaker PVC model, our techniques can achieve adaptive security under the standard model without random oracles.

Our main results and contributions are summarized below:

Definition of New Paradigm. We are the first ones to formally define signatures of correct computation (SCC) and its security and to study its relation to PVC.

Novel Constructions for Polynomial Manipulations. We focus on deriving efficient and optimized constructions for specific functionalities rather than generic constructions, as the approach taken by Parno et al. [31] and Canetti et al. [9]. We present efficient SCC constructions for expressive polynomial manipulations, including multivariate polynomial evaluation and differentiation. Operations on polynomials represent a common building block in a wide range of applications, such as in statistical analysis, scientific computing, and machine learning. Fiore and Gennaro [13] point out many interesting applications of publicly verifiable computation on polynomials, including its use in proofs of retrievability, verifiable keyword search, discrete Fourier tranform, and linear transformations. Our constructions are based on bilinear groups. We prove the adaptive security of our constructions under the random oracle model. 
Efficient Incremental Updates. Our constructions allow a trusted source to make incremental updates in time proportional to the number of the updated polynomial coefficients, and without performing a computation from scratch that would take linear time in the size of the polynomial.

Novel proof Techniques for Adaptive Security. Our constructions and proofs introduce several novel techniques. First, we observe key polynomial decomposition properties (Lemmas 1 and 3) that become the central idea underlying our constructions. Second, while achieving adaptive security appears relatively easy for univariate polynomial evaluation [23], achieving adaptive security in the multivariate case appears to be fundamentally more difficult. To this end, we present novel techniques that involve embedding randomness in the polynomial decomposition properties (Lemmas 2]and4), such that our simulator can later manipulate these random numbers in the proof. We give a high-level technical overview in Section 1.3

Contributions to Publicly Verifiable Computation. Our results also bring advances in the area of publicly verifiable computation. Specifically, our techniques can be readily applied to yield publicly verifiable computation schemes (for the same operations) with adaptive security (without the random oracle model) and with efficient updates. In comparison, existing PVC works [9]13]31], achieve adaptive security but do not support efficient updates. We give a more detailed comparison in Section 1.2 .

\subsection{Related Work}

Authenticated Data Structures. The SCC model is directly related to the model of authenticated data structures (ADS) [33]35]. In some sense, SCC and ADS are dual problems to each other, sharing exactly the same security properties. In SCC, a trusted source outsources a function, and a client wishes to verify the outcome of the function at a given point. In ADS, a trusted source outsources the data or a data structure, and the client wishes to verify the correctness of the result of a data structure query, e.g., dictionaries [18|26], graphs [20|25] and hash tables [29|34]. Most authenticated data structures schemes incur logarithmic or linear overheads for verification costs, with some exceptions being authenticated range queries [2[19] and set operations [30], where verification takes time proportional to the size of the answer.

Verifiable Computation in the Secret Key Setting (SVC). Recent works on verifiable computation [1|10|14] achieve efficient verification of general boolean circuits, but in the secret key model. Therefore they are inherently inadequate for the setting of signatures, which are required to be publicly verifiable.

Verifiable Computation for Polynomials. Benabbas et al. [3] developed methods for efficient verification of multivariate polynomial evaluation by using algebraic one-way functions-however, in the SVC model. This work does not achieve efficient updates of polynomial coefficients (specifically, in order to update a coefficient, one has to rerandomize all the existing coefficients), 1 Kate et al. [23] give a publicly verifiable commitment scheme for univariate polynomials, which is essentially an SCC scheme for

\footnotetext{
${ }^{1}$ However, apart from verification of polynomial evaluation, their techniques can be applied to
} support very efficient dynamic verifiable databases (constant query and update complexity). 
Table 1. Asymptotic cost on the client side. In the table below, $n$ is the number of variables in the polynomial and $d$ is the maximum degree. With SVC we denote a "secretly delegatable and verifiable scheme", with PVC we denote a "publicly delegatable and verifiable scheme", with PVC* we denote a "publicly verifiable but not publicly delegatable scheme" (see Section 1.2 Paragraph 5) and with SCC we denote a "signatures of correct computation scheme". Notice that an $n$-variate polynomial of degree $d$ can have up to $\left(\begin{array}{c}n+d \\ d\end{array}\right)$ terms, requiring up to $\left(\begin{array}{c}n+d \\ d\end{array}\right)$ time to evaluate. Therefore, the verification costs here are smaller than the cost of evaluating the polynomial. For PVC schemes, the client cost includes both delegation and verification costs.

\begin{tabular}{|l|l|l|c|c|l|}
\hline scheme & $\begin{array}{l}\text { polynomial } \\
\text { evaluation }\end{array}$ & $\begin{array}{l}\text { polynomial } \\
\text { differentiation }\end{array}$ & $\begin{array}{l}\text { efficient } \\
\text { updates }\end{array}$ & security & model \\
\hline \hline Benabbas et al. [3] & $n \log d$ & N/A & no & adaptive & SVC \\
\hline Parno et al. [31] & $n$ & $n+\log d$ & no & adaptive & PVC \\
\hline Canetti et al. [9] & polylog $\left(\left(\begin{array}{c}n+d \\
d\end{array}\right)\right)$ & polylog $\left(\left(\begin{array}{c}n+d \\
d\end{array}\right)\right)$ & no & adaptive & PVC \\
\hline Fiore and Gennaro [12[13] & $n \log d$ & N/A & no & adaptive & PVC* \\
\hline This paper & $n$ & $n+d$ & yes & selective & SCC \\
This paper & $n+d$ & $n+d^{2}$ & yes & adaptive & PVC \\
This paper & $n+d$ & $n+d^{2}$ & yes & adaptive (RO) & SCC \\
\hline
\end{tabular}

univariate polynomial evaluation. However, their scheme does not directly extend to multivariate polynomials. Also note that our construction is the first to support efficient verification of differentiation queries - even in the SVC setting.

Relation to CS Proofs and SNARGs. Our SCC model is strongly related to the model of computationally-sound proofs, introduced by Micali in 1994 [27], and to the subsequent works on succinct non-interactive arguments (SNARGs) by Groth [22], Bitansky et al. [45] and Gennaro et al. [15]. The main connection is that both SCC and SNARGs models are non-interactive and publicly verifiable (CS proofs can also be non-interactive in the random oracle model), i.e., a publicly verifiable proof can be computed independently from (and with no communication with) the verifier. We note here that all CS proofs and SNARGs constructions that have been presented in the literature are generalized, in that they can handle all of NP by using powerful tools such as the PCP theorem (with an exception of [15] that uses a different characterization of NP). Moreover, all of them (except for the work of Micali [27] that is secure in the random oracle model) are proved secure based on non-falsifiable assumptions [17], e.g., the works of Groth [22] and Gennaro et al. [15] use variants of the knowledge-based assumption introduced by Damgard [11]. Non-falsifiable assumptions are considered to be a lot stronger than all common assumptions used in cryptography (one-way functions, trapdoor permutations, DDH, RSA, LWE etc.). We note that the assumptions that we are using in our construction do not belong in this category-however, for verifying multivariate polynomials (not for univariate ones) we do use the random oracle, as the construction of Micali [27] does. The main difference (with [27]) however is that we do not use the PCP theorem, hence achieving more practical schemes.

Concurrent and Independent Works. Two closely related schemes are the ones by Parno et al. [31] and Cannetti et al. [9], which were developed concurrently with and independently from our work. 
In the PVC formulation proposed by Parno et al. [31], any client can verify that an untrusted server correctly computes a function $f$ on a specific input $\mathbf{a}$. Their definition however requires an input preparation randomized algorithm (ProbGen), mapping user inputs a to server inputs $\sigma_{\mathbf{a}}$ and preparing an object $\mathrm{VK}_{\mathbf{a}}$ to be used for verification, specific for $\sigma_{\mathbf{a}}$. Therefore, as opposed to the SCC setting, only the client that issued a query for a (or anyone who trusts this client) can verify that the server returned a valid signature (proof) for $f(\mathbf{a})$. For otherwise, a client running the ProbGen algorithm can potentially collude with the server to forge a proof, convincing another party to accept the proof. Apart from defining PVC, Parno et al. [31] give a construction for generalized boolean functions (closed under complement) from attribute-based encryption (ABE). Their construction is asymptotically efficient - the proof size is proportional to the size of the answer. Moreover, due to recent advances in ABE schemes by Lewko and Waters [24], the PVC constructions of Parno et al. [31] can be proved adaptively secure, since they directly inherit the security of the underlying ABE scheme.

A PVC scheme having similar properties with the scheme of Parno et al. [31] was presented by Canetti et al. [9], where client verification is polylogarithmic in the size of the evaluated circuit. Canetti et al. achieve adaptive security under a slightly weaker model (as Parno et al. point out [31]), in which the client needs to keep certain secret state. Their scheme shares the same limitation with the scheme of Parno et al. [31] in that a client can verify only his queries unless extra assumptions are put into place.

The most closely related works are the recent works by Fiore and Gennaro [13], who presented a PVC scheme tailored for multivariate polynomials that is based on algebraic one-way functions. An improved version [12] uses less complex assumptions such as RSA to achieve the same goal. The works by Fiore and Gennaro differ from ours in the following sense. First, they consider a model (denoted with PVC* in Table 1 ) that is more restrictive than the PVC model proposed by Parno et al. [31]-and hence more restrictive than the SCC model. Specifically, there is an explicit delegation phase where a problem instance is generated based on an input (as in the PVC definition by Parno et al. [31]). However, in their constructions (and unlike the original PVC definition), only the party who ran the setup algorithm for a specific function can run the problem generation algorithm. Therefore, their schemes are publicly verifiable, but not publicly delegatable. As a result, their schemes would not work for the application scenario where a pharmaceutical company outsources a genomic algorithm, and each user submits their own genomic data for computation. Moreover, they do not consider efficient updates of the polynomial coefficients. In comparison, their scheme has more efficient verification and a delegation step of $O(n \log d)$ cost. A detailed comparison of our scheme against several related works in terms of verification cost and security model is presented in Table 1

\subsection{Highlights of Techniques}

Multivariate Polynomial Evaluation. The polynomial commitment scheme by Kate et al. [23] can be employed to construct an SCC scheme of univariate polynomial evaluations. Specifically, Kate et al. [23] observe that to vouch for the outcome of a polynomial $f(x)$ in $\mathbb{Z}_{p}$ evaluated at the point $a \in \mathbb{Z}_{p}$, one can rely on the property that the polynomial $f(x)-f(a)$ is perfectly divisible by the degree- 1 polynomial 
$x-a$, where $a \in \mathbb{Z}_{p}$. In other words, one can find a polynomial $w(x)$ such that $f(x)-f(a)=(x-a) w(x)$. Using this property, they construct a witness from the term $w(x)$, and using the pairing operation in bilinear groups, they encode the above test $f(x)-f(a)=(x-a) w(x)$ in the exponents of group elements.

Unfortunately, the above test does not apply to the multivariate case. We therefore propose a novel technique based on the following observation. Let $f(\mathbf{x})$ be a multivariate polynomial in $\mathbb{Z}_{p}$ where $\mathbf{x}=\left[x_{1}, x_{2}, \ldots, x_{n}\right]$. Then, for $\mathbf{a}=\left[a_{1}, a_{2}, \ldots, a_{n}\right] \in \mathbb{Z}_{p}^{n}$, the polynomial $f(\mathbf{x})-f(\mathbf{a})$ can be expressed as $f(\mathbf{x})-f(\mathbf{a})=\sum_{i=1}^{n}\left(x_{i}-a_{i}\right) w_{i}(\mathbf{x})$. The polynomials $w_{i}(\mathbf{x})$ will be used to construct witnesses in our scheme. Specifically, we encode their terms as exponents of bilinear group elements. The verification is a pairing product equation encoding the above test in the exponent.

From Selective to Adaptive Security. The test that holds for the polynomial evaluation contains a sum of terms, as opposed to a single term in the univariate case [23]. This gives rise to certain technicalities in the proof, allowing us to prove only the weaker notion of selective security (see Definition 6 in the Appendix).

Going from selective security to adaptive security turns out to be non-trivial. To achieve this, we devise a novel technique where we build randomness into the polynomial decompositions (Lemmas 2 and 4) which are central to our constructions. As an immediate corollary of our adaptively secure SCC construction with random oracles, we construct an adaptively secure PVC scheme in the plain model.

Derivative Evaluation. A naive method to support verifiable derivative evaluation is for the source to commit to $n k$ polynomials during setup, corresponding to the 1 st, $2 \mathrm{nd}, \ldots, k$-th derivatives of each possible variable. However, as noted in Section 5 , this scheme results in increased setup and update overhead.

Our techniques for verifying the evaluation of an arbitrary derivative are inspired by the following observation that holds for first derivatives of univariate polynomials: Given a univariate polynomial $f(x)$, the remainder of dividing the polynomial $f(x)-f^{\prime}(a) x$ with the polynomial $(x-a)^{2}$ is always a constant polynomial, and not a degree-one polynomial, as would generally happen. In other words, $f(x)-f^{\prime}(a) x=$ $(x-a)^{2} q(x)+b$ for some $q(x) \in \mathbb{Z}_{p}[x]$, and $b \in \mathbb{Z}_{p}$. A similar, slightly more involved, observation can be made for higher-order derivatives and multivariate polynomials. More details are provided in Section 5 .

\section{Preliminaries, Definitions and Assumptions}

In this section, we give necessary definitions that are going to be used in the rest of the paper. The security parameter is denoted $\lambda$, PPT stands for probabilistic polynomialtime and $\operatorname{neg}(\lambda)$ denotes the set of negligible functions, i.e., all the functions less than $1 / p(\lambda)$, for all polynomials $p(\lambda)$. We also use bold letters for vector variables, i.e., $\mathbf{x}=\left[x_{1}, x_{2}, \ldots, x_{n}\right]$ denotes a vector of $n$ entries $x_{1}, x_{2}, \ldots, x_{n}$.

\subsection{Problem Definition}

We now formally define signatures of correct computation (SCC). 
Definition 1 (SCC scheme). An SCC scheme (signatures of correct computation) for a function family $\mathcal{F}$ is a tuple (KeyGen, Setup, Compute, Verify, Update) of five PPT algorithms with the following specification:

1. $(\mathrm{PK}, \mathrm{SK}) \leftarrow \operatorname{KeyGen}(\lambda, \mathcal{F}):$ Algorithm KeyGen takes as input the security parameter $\lambda$ and a function family $\mathcal{F}$. It outputs a public/secret key pair (PK, SK). KeyGen is run only once at system initialization by a trusted source;

2. $\mathrm{FK}(f) \leftarrow \operatorname{Setup}(\mathrm{SK}, \mathrm{PK}, f):$ Algorithm Setup (run by a trusted source) takes as input the secret key SK, the public key $\mathrm{PK}$, and a function $f \in \mathcal{F}$. It outputs the function public key $\mathrm{FK}(f)$ for the function $f$;

3. $(v, w) \leftarrow$ Compute(PK, $f, \mathbf{a}):$ Algorithm Compute (run by an untrusted server) takes as input the public key $\mathrm{PK}$, a function $f \in \mathcal{F}$ and a value $\mathbf{a} \in \operatorname{domain}(f)$. It outputs a pair $(v, w)$, where $v=f(\mathbf{a})$ and $w$ is a signature;

4. $\{0,1\} \leftarrow$ Verify $(\mathrm{PK}, \mathrm{FK}(f)$, a, $v, w)$ : Algorithm Verify (run by any verifier) takes as input the public key $\mathrm{PK}$, the function public key $\mathrm{FK}(f)$, value $\mathbf{a} \in \operatorname{domain}(f)$, a claimed result $v$ and a signature $w$. It outputs 0 or 1 ;

5. $\mathrm{FK}\left(f^{\prime}\right) \leftarrow$ Update(SK, PK, $\left.\mathrm{FK}(f), f^{\prime}\right)$ : Algorithm Update (run by a trusted source) takes as input the secret key SK, the public key PK, the function public key $\mathrm{FK}(f)$ for the old function $f$ and the updated function description $f^{\prime}$. It outputs the updated function public key $\mathrm{FK}\left(f^{\prime}\right)$.

The Update algorithm allows the source to update the function $f$ to a new function $f^{\prime}$. A naive way to implement Update is to simply run the Setup algorithm again for the new $f^{\prime}$. However, in practice, one may wish to allow more efficient incremental updates (and this is what is achieved by our constructions).

\subsection{Correctness and Security Definitions}

We describe now the correctness and adaptive security definitions for SCC. Intuitively, an SCC scheme is correct if whenever its algorithms are executed honestly, it never rejects a correct signature. Also, it is secure if, after the setup/update algorithms have been executed, an adversary cannot convince a verifier to accept a wrong result on an input of his choice, except with negligible probability.

Definition 2 (Correctness of an SCC scheme). Let $\lambda$ be the security parameter and let $\mathcal{P}$ be an SCC scheme (KeyGen, Setup, Compute, Verify, Update) for a function family $\mathcal{F}$. Let $(\mathrm{PK}, \mathrm{SK}) \leftarrow \operatorname{KeyGen}(\lambda, \mathcal{F})$. For all $i=1, \ldots$, poly $(\lambda)$, for any function $f_{i} \in \mathcal{F}$, suppose $\mathrm{FK}\left(f_{i}\right)$ is the output of Update(SK, PK, $\left.\mathrm{FK}\left(f_{i-1}\right), f_{i}\right)$, where $\mathrm{FK}\left(f_{0}\right)$ is output by algorithm $\operatorname{Setup}\left(\mathrm{SK}, \mathrm{PK}, f_{0}\right)$ for some $f_{0} \in \mathcal{F}$. We say that $\mathcal{P}$ is correct, if for any $i=0, \ldots$, poly $(\lambda)$, for any $\mathbf{a} \in \operatorname{domain}\left(f_{i}\right)$, it is $1 \leftarrow \operatorname{Verify}\left(\operatorname{PK}, \operatorname{FK}\left(f_{i}\right), \mathbf{a}, v, w\right)$, where $(v, w) \leftarrow$ Compute $\left(\mathrm{PK}, f_{i}, \mathbf{a}\right)$.

Definition 3 (Adaptive security of an SCC scheme). Let $\lambda$ be the security parameter and let $\mathcal{P}$ be an SCC scheme (KeyGen, Setup, Compute, Verify, Update) for a function family $\mathcal{F}$. We say that $\mathcal{P}$ is adaptively secure if no PPT adversary $\mathcal{A}$ has more than negligible probability neg $(\lambda)$ in winning the following security game, played between the adversary $\mathcal{A}$ and a challenger: 
1. Initialization. The challenger runs algorithm KeyGen which outputs (PK, SK) and then gives $\mathrm{PK}$ to the adversary but maintains SK secret;

2. Setup and update. The adversary makes an oracle query to the $\operatorname{Setup}\left(\mathrm{SK}, \mathrm{PK}, f_{0}\right)$ algorithm, specifying an initial function $f_{0} \in \mathcal{F}$, outputting $\mathrm{FK}\left(f_{0}\right)$. Then, for $i=1, \ldots, k$, where $k=$ poly $(\lambda)$, he makes a polynomial number of oracle queries to the Update(SK, PK, $\left.\mathrm{FK}\left(f_{i-1}\right), f_{i}\right)$ algorithm, each time specifying $f_{i} \in \mathcal{F}$. The challenger answers the queries by returning the resulting $\mathrm{FK}\left(f_{i}\right)$;

3. Forgery. The adversary $\mathcal{A}$ outputs a point $\mathbf{b} \in \operatorname{domain}\left(f_{i}\right)$ for some $0 \leq i \leq k$, and the forgery $(\mathbf{b}, v, w)$.

The adversary $\mathcal{A}$ wins the game if $1 \leftarrow \operatorname{Verify}\left(\mathrm{PK}, \mathrm{FK}\left(f_{i}\right), \mathbf{b}, v, w\right)$ and $f_{i}(\mathbf{b}) \neq v$.

\subsection{SCC Implies PVC}

As we highlighted in the introduction, signatures of correct computation (SCC) are stronger than the publicly verifiable computation (PVC) notions studied in concurrent but independent papers [9 12 13 131 ]. Specifically, a correct and secure SCC scheme implies a correct and secure PVC scheme, but not the other way around. To see that, one can implement algorithm $\sigma_{\mathbf{a}} \leftarrow \operatorname{ProbGen}(\mathrm{PK}, \mathbf{a})$ of the PVC scheme (e.g., [31]) to simply output a and all the other algorithms remain the same.

For completeness, in Definition 6 in the Appendix, we also provide the definition of publicly verifiable computation (PVC) . Our PVC definition is essentially equivalent to those proposed by Parno et al. [31] and Canetti et al. [9], with the exception that we augment it with an Update algorithm which a trusted source can employ to incrementally update the outsourced function (also, our ProbGen algorithm is called Challenge).

\subsection{Multivariate Polynomials Notation}

We now give some notation for multivariate polynomials. We use the notion of a multiset over some universe $\mathcal{U}$, a generalized set comprising elements from the universe $\mathcal{U}$, where each element can appear more than once; for example, $\{1,1,2,3,3,3\}$ is a multiset. In this paper, we use the following notation to denote multisets. Formally, a multiset $S: \mathcal{U} \rightarrow \mathbb{Z}^{\geq 0}$ is a function mapping each element in a universe $\mathcal{U}$ to its multiplicity. For any $x \notin S, S(x)=0$. E.g., for the multiset $\{a, a, b, c, c, c\}$, we have $S(a)=2$, $S(b)=1, S(c)=3$; however, $S(e)=0$ since $e$ is not contained in the above multiset.

Let now $S, T$ denote two multisets over universe $\mathcal{U}$. It is $S \subseteq T$, if $\forall a \in \mathcal{U}$, $S(a) \leq T(a)$. The size of $S$ over universe $\mathcal{U}$, denoted $|S|$, is defined as the sum of the multiplicity of all elements in $S$, i.e., $|S|=\sum_{a \in \mathcal{U}} S(a)$. Finally, $\mathcal{S}_{d, n}$ denotes the set of multisets of size at most $d$ over the universe $\{1,2, \ldots, n\}$. Let now $f \in \mathbb{Z}_{p}\left[x_{1}, x_{2}, \ldots, x_{n}\right]=\mathbb{Z}_{p}[\mathbf{x}]$ be an $n$-variate polynomial over $\mathbb{Z}_{p}$ with maximum degree $d$. We can use the following generic notation to represent $f$, i.e.,

$$
f(\mathbf{x})=f\left(x_{1}, x_{2}, \ldots, x_{n}\right)=\sum_{S \in \mathcal{S}_{d, n}} c_{S} \cdot \prod_{i \in S} x_{i}^{S(i)} .
$$

For example, the multiset $\{1,1,2,2,2,5\}$ corresponds to the term for $x_{1}^{2} x_{2}^{3} x_{5}$ in the expanded form of the polynomial. The empty multiset $\emptyset$ corresponds to the constant 
term in the polynomial. Finally, the degree of a multivariate polynomial is the maximum total degree of any monomial contained in the polynomial. For example, the degree of the polynomial $3 x_{1} x_{2}+x_{3}^{3} x_{4} x_{5}$ is 5 .

\subsection{Bilinear Groups and Computational Assumption}

We now review some background on bilinear groups of prime order. Let $\mathbb{G}$ be a cyclic multiplicative group of prime order $p$, generated by $g$. Let also $\mathbb{G}_{T}$ be a cyclic multiplicative group with the same order $p$ and e $: \mathbb{G} \times \mathbb{G} \rightarrow \mathbb{G}_{T}$ be a bilinear pairing with the following properties: (1) Bilinearity: $\mathrm{e}\left(P^{a}, Q^{b}\right)=\mathrm{e}(P, Q)^{a b}$ for all $P, Q \in \mathbb{G}$ and $a, b \in \mathbb{Z}_{p}$; (2) Non-degeneracy: $\mathrm{e}(g, g) \neq 1$; (3) Computability: There is an efficient algorithm to compute $e(P, Q)$ for all $P, Q \in \mathbb{G}$. We denote with $\left(p, \mathbb{G}, \mathbb{G}_{T}, \mathrm{e}, g\right)$ the bilinear pairings parameters, output by a PPT algorithm on input $1^{\lambda}$. We use the following computational assumption [6]:

Definition 4 (Bilinear $\ell$-strong Diffie-Hellman assumption). Suppose $\lambda$ is the security parameter and let $\left(p, \mathbb{G}, \mathbb{G}_{T}, e, g\right)$ be a uniformly randomly generated tuple of bilinear pairings parameters. Given the elements $g, g^{t}, \ldots, g^{t^{\ell}} \in \mathbb{G}$ for some $t$ chosen at random from $\mathbb{Z}_{p}^{*}$, for $\ell=$ poly $(\lambda)$, there is no PPT algorithm that can output the pair $\left(c, \mathrm{e}(g, g)^{1 /(t+c)}\right) \in \mathbb{Z}_{p}^{*} \backslash\{-t\} \times \mathbb{G}_{T}$ except with negligible probability $\mathrm{neg}(\lambda)$.

\section{Selectively Secure Multivariate Polynomial Evaluation}

As a warm-up exercise, in this section we first present an SCC scheme for multivariate polynomial evaluation that is secure under a relaxed security model, namely, the selective security model. Then, in Section 4, we explain how to augment this selectively secure scheme and achieve adaptive security in the random oracle model.

Selective security is weaker than adaptive security, requiring the adversary to commit ahead of time to the challenge point $\mathbf{a}$, which is analogous to the selective security notion often adopted in Identity-Based Encryption (IBE) [7], Attribute-Based Encryption (ABE) [21], Functional Encryption (FE) [32] and Predicate Encryption (PE) [8]. The detailed selective security definition is described in Definition 6in the Appendix.

\subsection{Intuition}

Our construction relies on the following key observation.

Lemma 1 (Polynomial decomposition). Let $f(\mathbf{x}) \in \mathbb{Z}_{p}[\mathbf{x}]$ be an $n$-variate polynomial. For all $\mathbf{a} \in \mathbb{Z}_{p}^{n}$, there exist polynomials $q_{i}(\mathbf{x}) \in \mathbb{Z}_{p}[\mathbf{x}]$ such that the polynomial $f(\mathbf{x})-f(\mathbf{a})$ can be expressed as $f(\mathbf{x})-f(\mathbf{a})=\sum_{i=1}^{n}\left(x_{i}-a_{i}\right) q_{i}(\mathbf{x})$. Moreover, there exists a polynomial-time algorithm to find the above polynomials $q_{i}(\mathbf{x})$.

The above lemma can be proved by explicit construction, dividing each time the polynomial $f(\mathbf{x})-f(\mathbf{a})$ with $\left(x_{i}-a_{i}\right)$. Its proof is given in the full version of our paper [28].

Given now an $n$-variate polynomial $f(\mathbf{x})$, the trusted source runs algorithms KeyGen and Setup to create the function public key $\mathrm{FK}(f)=g^{f(\mathbf{t})}$ of the polynomial $f$ 
evaluated over a randomly chosen point $\mathbf{t}$. Later in the computation stage, when a server wishes to prove that $v$ is indeed the value $f(\mathbf{a})$, it will rely on the key observation stated in Lemma 1, It will compute $n$ polynomials $q_{1}(\mathbf{x}), q_{2}(\mathbf{x}), \ldots, q_{n}(\mathbf{x})$ such that the relation of Lemma 1 holds, and the values $g^{q_{i}(\mathbf{t})}(i=1, \ldots, n)$ will be provided as the signature. To allow the server to evaluate the polynomials $q_{i}(\mathbf{x})$ at the commitment point $\mathbf{t}$ in the exponent, the public key must contain appropriate helper terms. If the claimed computation result $v$ is correct, then the following must be true, where both sides of the equation are evaluated at the commitment point $\mathbf{t}$, i.e., it should be $f(\mathbf{t})-v=\sum_{i \in[n]}\left(t_{i}-a_{i}\right) q_{i}(\mathbf{t})$. We note here that in the real construction, the terms in the above equation are encoded in the exponents of group elements, and therefore the verifier cannot directly check the above equation. However, the verifier can check the above condition using operations in the bilinear group, including the pairing operation which allows one to express one multiplication in the exponent. The bilinear group operations directly translate to checking the above condition in the exponent.

\subsection{Detailed Construction}

We now present our selectively secure SCC scheme supporting multivariate polynomial evaluation.

$\operatorname{Algorithm}(\mathrm{PK}, \mathrm{SK}) \leftarrow \operatorname{KeyGen}(\lambda, \mathcal{F})$ : Suppose that the function family $\mathcal{F} \subseteq \mathbb{Z}_{p}[\mathbf{x}]$ represents all polynomials over $\mathbb{Z}_{p}$ with at most $n$ variables and degree bounded by $d$. Namely, family $\mathcal{F}$ contains the polynomials represented by multisets in set $\mathcal{S}_{n, d}$ (see Equation 2.1). The KeyGen algorithm invokes the bilinear group generation algorithm to generate a bilinear group instance of prime order $p$ (of $\lambda$ bits), with a bilinear map function e : $\mathbb{G} \times \mathbb{G} \rightarrow \mathbb{G}_{T}$. Then it chooses a random generator $g \in \mathbb{G}$ and a random point $\mathbf{t}=\left[t_{1}, t_{2}, \ldots, t_{n}\right] \in \mathbb{Z}_{p}^{n}$ and computes the signature generation set $\mathcal{W}_{n, d}$

$$
\mathcal{W}_{n, d}=\left\{g^{\prod_{i \in S} t_{i}^{S(i)}}: \forall S \in \mathcal{S}_{n, d}\right\} .
$$

For example, $\mathcal{W}_{2,2}$ contains the elements $g, g^{t_{1}}, g^{t_{2}}, g^{t_{1}^{2}}, g^{t_{2}^{2}}, g^{t_{1} t_{2}^{2}}, g^{t_{1}^{2} t_{2}}, g^{t_{1}^{2} t_{2}^{2}}$. The algorithm finally outputs the public key PK that contains $g, \mathcal{W}_{n, d}$ and the description of $\mathbb{G}, \mathbb{G}_{T}$, e. The secret key SK contains the commitment point $\mathbf{t}$. We describe an optimization referring to reducing the number of group elements of $\mathcal{W}_{n, d}$ in the full version of the paper [28].

Algorithm $\mathrm{FK}(f) \leftarrow \operatorname{Setup}(\mathrm{SK}, \mathrm{PK}, f)$ : Let $f(\mathbf{x}) \in \mathbb{Z}_{p}[\mathbf{x}]$ denote an $n$-variate polynomial of maximum degree $d$ over $\mathbb{Z}_{p}$ that is represented by the multisets $S_{1}, S_{2}, \ldots, S_{k} \in$ $\mathcal{S}_{n, d}$ and the respective coefficients $c_{1}, c_{2}, \ldots, c_{k} \in \mathbb{Z}_{p}$ (the polynomial has $k$ terms), as defined in Equation 2.1. The setup algorithm, by using the signature generation set $\mathcal{W}_{n, d}$ contained in $\mathrm{PK}$, computes the polynomial public key, i.e.,

$$
\mathrm{FK}(f)=g^{f(\mathbf{t})}=\left(g^{\prod_{i \in S_{1}} t_{i}^{S_{1}(i)}}\right)^{c_{1}} \times\left(g^{\prod_{i \in S_{2}} t_{i}^{S_{2}(i)}}\right)^{c_{2}} \times \ldots \times\left(g^{\prod_{i \in S_{k}} t_{i}^{S_{k}(i)}}\right)^{c_{k}} .
$$

The algorithm outputs the function public key $\mathrm{FK}(f)$.

Algorithm $(v, w) \leftarrow$ Compute $(\mathrm{PK}, f, \mathbf{a})$ : This algorithm first computes $v=f(\mathbf{a})$. Using Lemma 1, it finds an appropriate set of polynomials $q_{1}(\mathbf{x}), q_{2}(\mathbf{x}), \ldots, q_{n}(\mathbf{x})$ to 
express polynomial $f(\mathbf{x})-v$ as $f(\mathbf{x})-v=\sum_{i=1}^{n}\left(x_{i}-a_{i}\right) q_{i}(\mathbf{x})$. The signature $w$ is a vector of $n$ witnesses $w_{1}, w_{2}, \ldots, w_{n}$, such that $w_{i}=g^{q_{i}(\mathbf{t})}$ for all $i \in[n]$. Note that $w_{i}$ can easily be computed using the signature generation set $\mathcal{W}_{n, d}$, as is achieved for the function public key in Equation 3.3 It finally outputs the pair $(v, w)$, where $v$ is the outcome of the polynomial evaluated at $\mathbf{a}$, and $w$ is the signature of correctness.

Algorithm Verify $(\mathrm{PK}, \mathrm{FK}(f), \mathbf{a}, v, w)$ : Parse $\mathrm{PK}$ as the signature generation set $\mathcal{W}_{n, d}$. To verify that $v$ is indeed $f(\mathbf{a})$, given a signature $w=\left[w_{1}, w_{2}, \ldots, w_{n}\right]$, algorithm Verify checks if the following equation holds:

$$
\mathrm{e}\left(\mathrm{FK}(f) g^{-v}, g\right) \stackrel{?}{=} \prod_{i=1}^{n} \mathrm{e}\left(g^{t_{i}-a_{i}}, w_{i}\right)
$$

In the above, the terms $g^{t_{i}}$ are contained in PK (specifically in $\mathcal{W}_{n, d}$ ) and the function public key $\mathrm{FK}(f)$ equals $g^{f(\mathbf{t})}$. The algorithm accepts the result $v$, and outputs 1 if the above equations hold; otherwise, it rejects and outputs 0 .

Algorithm $\mathrm{FK}\left(f^{\prime}\right) \leftarrow \operatorname{Update}\left(\mathrm{SK}, \mathrm{PK}, \mathrm{FK}(f), f^{\prime}\right)$ : Let $f$ denote the current polynomial and $f^{\prime}$ be the new polynomial that corresponds to the update. Assume $f^{\prime}$ and $f$ differ in only one coefficient. Specifically, let $S$ denote the multiset corresponding to that coefficient 2 Suppose the current function public key is $\mathrm{FK}(f)$. The algorithm sets

$$
\mathrm{FK}\left(f^{\prime}\right)=\mathrm{FK}(f) \cdot g^{\left(c_{S}^{\prime}-c_{S}\right)} \prod_{i \in S} t_{i}^{S(i)},
$$

updating $\mathrm{FK}(f)$ to $\mathrm{FK}\left(f^{\prime}\right)$, the new function public key. We now state our first theorem.

Theorem 1. There exists an SCC scheme for polynomial evaluation such that (1) It is correct according to Definition 2. (2) For univariate polynomials, it is adaptively secure according to Definition 3 and under the $\ell-S B D H$ assumption; (3) For multivariate polynomials, it is selectively secure according to Definition 6 and under the $\ell-S B D H$ assumption.

The correctness of our construction follows in a straightforward manner from Lemma1, and the bilinear property of the pairing operation e. The asymptotic cost analysis of the scheme's algorithms are presented in Section 6, The security proofs are presented in the full version of the paper [28]. However, we give a proof sketch in the following.

\subsection{Selective Security Proof Sketch}

We briefly explain the selective security proof intuition of our scheme. The simulator obtains an $\ell$-SBDH instance, $g, g^{\tau}, \ldots, g^{\tau^{\ell}} \in \mathbb{G}$ and it will construct a simulation such that if an adversary can break the selective security of the SCC scheme, the simulator can leverage it to break the $\ell-\mathrm{SBDH}$ instance. Specifically, with knowledge of the challenge point $\mathbf{a}=\left[a_{1}, a_{2}, \ldots, a_{n}\right]$ that the adversary commits to at the beginning of the selective security game, the simulator can carefully craft the simulation such that

\footnotetext{
${ }^{2}$ I.e., the only difference between $f$ and $f^{\prime}$ is that the coefficient $c_{S}$ corresponding to term $\prod_{i \in S} x_{i}^{S(i)}$ is updated to $c_{S}^{\prime}$ in $f^{\prime}$.
} 
$t_{i}-a_{i}=\lambda_{i}(\tau+c)$, where $t=\left[t_{1}, t_{2}, \ldots, t_{n}\right]$ represents the committed point used to compute the polynomial digest, and $\lambda_{i}$ and $c$ are constants known to the simulator.

If an adversary can forge a signature for a wrong outcome of a polynomial, then the simulator is able to raise terms in Equation 3.4 to the $(\tau+c)^{-1}$ power and output $\mathrm{e}(g, g)^{(\tau+c)^{-1}}$, breaking in this way the $\ell$-SBDH assumption. Notice that in the selective security proof, the simulator's ability to take appropriate terms in Equation 3.4 to the $(\tau+c)^{-1}$ power relies on knowing the challenge point $\mathbf{a}$ in advance, and the ability to craft the simulation such that $t_{i}-a_{i}=\lambda_{i}(\tau+c)$.

\section{Adaptively Secure Multivariate Polynomial Evaluation}

In this section, we augment the above selectively secure SCC scheme to achieve adaptive security in the random oracle model. We also show that the same techniques can be applied to construct an adaptively secure PVC scheme under the formulation of Parno et al. [31] without the random oracle model.

\subsection{Intuition}

The intuition of the new construction is similar to the selectively secure construction. For technical reasons explained later, instead of relying on the polynomial decomposition method described in Lemma1, we use a new decomposition that is randomized, so that it can later be manipulated by a simulator in the proof to achieve adaptive security. The decomposition we are using is the following:

Lemma 2 (Randomized decomposition). Let $f(\mathbf{x}) \in \mathbb{Z}_{p}[\mathbf{x}]$ be an n-variate polynomial of degree at most $d$. For all $\mathbf{a} \in \mathbb{Z}_{p}^{n}$ and for all $r_{1}, \ldots, r_{n-1} \in \mathbb{Z}_{p}$ such that $r_{1} r_{2} \ldots r_{n-1} \neq 0$, there exist polynomials $q_{i}(\mathbf{x}) \in \mathbb{Z}_{p}[\mathbf{x}]$ such that the polynomial $f(\mathbf{x})-f(\mathbf{a})$ can be expressed as

$$
f(\mathbf{x})-f(\mathbf{a})=\sum_{i=1}^{n-1}\left[r_{i}\left(x_{i}-a_{i}\right)+x_{i+1}-a_{i+1}\right] q_{i}(\mathbf{x})+\left(x_{n}-a_{n}\right) q_{n}\left(x_{n}\right)
$$

where $q_{n}\left(x_{n}\right)$ is a polynomial of degree at most $d$ that contains only variable $x_{n}$. Moreover, there exists a polynomial-time algorithm to find the above polynomials $q_{i}(\mathbf{x})$.

The above lemma can be proved by explicit construction, each time dividing the polynomial by $r_{i}\left(x_{i}-a_{i}\right)+x_{i+1}-a_{i+1}$, for increasing values of $i$, in a way such that the remainder should not contain $x_{i}$. The full proof of Lemma2 is provided in the full version of our paper [28]. We note here that in our construction explained below, the numbers $r_{1}, r_{2}, \ldots, r_{n-1}$ mentioned in Lemma2 will be chosen "at random" by calling a hash function modelled as a random oracle (see Equation 4.5).

\subsection{Detailed Construction}

We now continue with the algorithms of our adaptively secure SCC scheme. 
$\operatorname{Algorithm}(\mathrm{PK}, \mathrm{SK}) \leftarrow \operatorname{KeyGen}(\lambda, \mathcal{F}):$ Same as in Section 3

Algorithm $\mathrm{FK}(f) \leftarrow \operatorname{Setup}(\mathrm{SK}, \mathrm{PK}, f)$ : Same as in Section 3 .

$\operatorname{Algorithm}(v, w) \leftarrow$ Compute $(\mathrm{PK}, f, \mathbf{a})$ : Parse a as $\left[a_{1}, a_{2}, \ldots, a_{n}\right]$. The algorithm first computes the outcome of the polynomial $v=f(\mathbf{a})$. Next, compute the following, where $\mathrm{H}:\{0,1\}^{*} \rightarrow \mathbb{Z}_{p}$ is a hash function (later modelled as a random oracle):

$$
\forall 1 \leq i \leq n-1: \quad r_{i}=\mathrm{H}(\mathbf{a} \| i) .
$$

Now, using Lemma2 find an appropriate set of polynomials $q_{1}(\mathbf{t}), q_{2}(\mathbf{t}), \ldots, q_{n}\left(t_{n}\right)$ to express polynomial $f(\mathbf{x})-f(\mathbf{a})$ as $\sum_{i=1}^{n-1}\left[r_{i}\left(x_{i}-a_{i}\right)+x_{i+1}-a_{i+1}\right] q_{i}(\mathbf{x})+\left(x_{n}-\right.$ $\left.a_{n}\right) q_{n}\left(x_{n}\right)$. Next, leverage the signature generation set $\mathcal{W}_{n, d}$ (see Equation 3.2) to compute $w_{i}=g^{q_{i}(\mathbf{t})}$ for $1 \leq i \leq n-1$. It is not hard to see that all $w_{i}$ 's can be computed from $\mathcal{W}_{n, d}$. The signature $w$ is composed as $w=\left[w_{1}, w_{2}, \ldots, w_{n}\right.$, polynomial $\left.q_{n}\left(x_{n}\right)\right]$, where the polynomial $q_{n}\left(x_{n}\right)$ contains the description of the polynomial, i.e., up to $d$ coefficients $\beta_{d}, \ldots, \beta_{0}$, since it is a univariate polynomial in $x_{n}$ of degree at most $d$.

The algorithm outputs the pair $(v, w)$ denoting the outcome of the polynomial evaluated at $\mathbf{a}$, and a signature to vouch for the correctness of the computation.

Algorithm $\{0,1\} \leftarrow \operatorname{Verify}(\mathrm{PK}, \mathrm{FK}(f), \mathbf{a}, v, w):$ Parse a as $\left[a_{1}, a_{2}, \ldots, a_{n}\right] \in \mathbb{Z}_{p}^{n}$; then parse the signature $w$ as $\left[w_{1}, w_{2}, \ldots, w_{n-1}\right.$, polynomial $\left.q_{n}\left(x_{n}\right)\right]$. To verify that $v$ is indeed the outcome of the correct polynomial evaluated at point $\mathbf{a} \in \mathbb{Z}_{p}^{n}$, algorithm Verify first computes $g^{q_{n}\left(t_{n}\right)}$ using the signature generation set $\mathcal{W}_{n, d}$ (Equation 3.2) which is part of the public key PK.

Next, it computes the $r_{i}$ values in the same way as in Equation 4.5, namely, $r_{i}=$ $\mathrm{H}(\mathbf{a} \| i)$ for $1 \leq i \leq n-1$. Finally, it checks if the following equation holds:

$$
\mathrm{e}\left(\mathrm{FK}(f) \cdot g^{-v}, g\right) \stackrel{?}{=} \prod_{i=1}^{n-1} \mathrm{e}\left(g^{r_{i}\left(t_{i}-a_{i}\right)+t_{i+1}-a_{i+1}}, w_{i}\right) \text { e }\left(g^{t_{n}-a_{n}}, g^{q_{n}\left(t_{n}\right)}\right)
$$

In the above, the terms $g^{t_{i}}$ are contained in PK (specifically in $\mathcal{W}_{n, d}$ ) and FK $(f)$ equals $g^{f(\mathbf{t})}$. The algorithm accepts if the above equation holds; otherwise, it rejects.

Algorithm $\mathrm{FK}\left(f^{\prime}\right) \leftarrow$ Update $\left(\mathrm{SK}, \mathrm{PK}, \mathrm{FK}(f), f^{\prime}\right)$ : Same as in Section 3 .

\subsection{Adaptive Security Proof Sketch}

The simulator obtains an $\ell$-SBDH instance, $g, g^{\tau}, \ldots, g^{\tau^{\ell}} \in \mathbb{G}$ and it will construct a simulation such that if an adversary can break the adaptive security of the SCC scheme, the simulator can leverage it to break the $\ell-\mathrm{SBDH}$ instance. Unlike in the selective security proof of Section 3.3 , without the adversary committing to the challenge point in advance, the simulator cannot craft terms to satisfy conditions such as $t_{i}-a_{i}=$ $\lambda_{i}(t+c)$ - but this condition is crucial later for the simulator to compute $\mathrm{e}(g, g)^{(\tau+c)^{-1}}$ and break the hardness assumption.

To circumvent this barrier in the proof, we embed "randomness" into the verification equation, such that the simulator can manipulate these random numbers to satisfy a condition described below, without having to know the challenge point ahead of time: 


$$
r_{i}\left(t_{i}-a_{i}\right)+t_{i+1}-a_{i+1}=\lambda_{i}(\tau+c) \text { for } i=1, \ldots, n-1,
$$

where $\lambda_{i}$ and $c$ are constants known to the simulator.

Specifically, since these random numbers are outputs from a "random" hash function, under the random oracle model, the simulator can manipulate the answers to the random oracle queries in the simulation to achieve the above goal. Note that our SCC signature with adaptive security has size $O(n+d)$, as opposed to $O(n)$, which was the size of the signature in the selectively secure scheme (see Section 6). This is because it is essential the signature contain the polynomial $q_{n}\left(x_{n}\right)$ for the adaptive security proof to work, so that the simulator can divide both sides of Equation 4.6 with $\tau+c$. We can now state our main theorem (see detailed proof in the full version of our paper [28]).

Theorem 2. There exists an SCC scheme for the evaluation of multivariate polynomials such that (1) It is correct according to Definition 2. (2) It is adaptively secure according to Definition 3 under the $\ell-S B D H$ assumption and in the random oracle model.

\subsection{An Adaptively Secure PVC Scheme without Random Oracles}

Our techniques can be readily adapted to construct an adaptively secure PVC scheme for multivariate polynomial evaluation-see Section 2.3. Neverthelsess, if we were to use the observations of Section 2.3 as a black box, we would construct a PVC scheme that has the random oracle. However, we are able to remove the random oracle by taking advantage of the fact that PVC is weaker than SCC.

The resulting PVC scheme is very similar to our construction in this section-except that in the PVC scheme, the random numbers $r_{i}$ 's are directly chosen at random (as a challenge) by a client issuing a query to the untrusted server, instead of being the outputs of a hash function modeled as a random oracle. We provide the detailed PVC scheme with full security in the the full version of the paper [28].

Theorem 3. There exists a PVC scheme for the evaluation of multivariate polynomials of total such that (1) It is correct according to Definition 8. (2) It is adaptively secure according to Definition 9 and under the $\ell-S B D H$ assumption.

\section{SCC Schemes for Polynomial Differentiation}

In this section, we construct an SCC scheme for the verification of differentiation queries. Given a multivariate polynomial $f(\mathbf{x})$, we show how to construct signatures of correct computation for derivatives $\partial^{k} f(\mathbf{x}) / \partial x_{j}^{k}(\mathbf{a})$ evaluated at a chosen point $\mathbf{a}$.

One naive method to support verification of derivative computation is to commit to all $n k$ polynomials corresponding to all the possible derivatives ( $k$ in total) of each possible variable. This would incur a setup cost of $O\left(n k\left(\begin{array}{c}n+d \\ d\end{array}\right)\right)$. In contrast, our construction requires only $O\left(\left(\begin{array}{c}n+d \\ d\end{array}\right)\right)$ setup cost (see Section 6), the same with the polynomial evaluation scheme. Another drawback of the naive method is increased update cost, since an update operation would now involve updating all $n k$ polynomials. In contrast, our construction allows for efficient incremental updates. 


\subsection{Intuition}

The intuition of supporting polynomial differentiation is similar to the evaluation case. In place of the decomposition lemmas (Lemmas 1 and 2) for polynomial evaluation, we have the following counterparts (Lemmas 3 and 4) for derivative computation:

Lemma 3 (Decomposition for derivatives). For a $\in \mathbb{Z}_{p}^{n}$, the n-variate polynomial $f(\mathbf{x}) \in \mathbb{Z}_{p}[\mathbf{x}]$ can be expressed as

$$
f(\mathbf{x})=\sum_{i=1}^{n-1}\left(x_{i}-a_{i}\right) u_{i}(\mathbf{x})+\left(x_{n}-a_{n}\right)^{k+1} q\left(x_{n}\right)+c_{k} x_{n}^{k}+\ldots+c_{1} x_{n}+c_{0} .
$$

Then, the $k$-th derivative of $f(\mathbf{x})$ wrt $x_{n}$ equals $k ! \cdot c_{k}$ at point $\mathbf{a}$, i.e., $\partial^{k} f(\mathbf{x}) / \partial x_{n}^{k}(\mathbf{a})=$ $k ! \cdot c_{k}$. A similar result holds for other variables $x_{i}$ by variable renaming.

Lemma 4 (Randomized decomposition for derivatives). For a $\in \mathbb{Z}_{p}^{n}$ and for all $r_{1}, \ldots, r_{n-2} \in \mathbb{Z}_{p}$ such that $r_{1} r_{2} \ldots r_{n-2} \neq 0$, the $n$-variate polynomial $f(\mathbf{x}) \in \mathbb{Z}_{p}[\mathbf{x}]$ can be expressed as

$$
\begin{aligned}
f(\mathbf{x}) & =\sum_{i=1}^{n-2}\left[r_{i}\left(x_{i}-a_{i}\right)+x_{i+1}-a_{i+1}\right] u_{i}(\mathbf{x})+\left(x_{n-1}-a_{n-1}\right) u_{n-1}(\mathbf{x}) \\
& +\left(x_{n}-a_{n}\right)^{k+1} q\left(x_{n}\right)+c_{k} x_{n}^{k}+\sum_{i=0}^{k} c_{i} x_{n}^{i},
\end{aligned}
$$

where $u_{n-1}(\mathbf{x})$ is a polynomial containing only variables $x_{n-1}$ and $x_{n}$ and $q\left(x_{n}\right)$ is a polynomial containing only variable $x_{n}$. Then, the $k$-th derivative of $f(\mathbf{x})$ wrt $x_{n}$ equals $k ! \cdot c_{k}$ at point $\mathbf{a}$, i.e., $\partial^{k} f(\mathbf{x}) / \partial x_{n}^{k}(\mathbf{a})=k ! \cdot c_{k}$. A similar result holds for other variables $x_{i}$ by variable renaming.

Similar to the multivariate polynomial evaluation case, Lemmas 3 and 4 allow us to construct respectively: 1) an SCC scheme for polynomial differentiation with selective security; and 2) an SCC scheme for polynomial differentiation with adaptive security in the random oracle model and a PVC scheme for polynomial differentiation with adaptive security without the random oracle model .

\subsection{Detailed Construction}

We now present the adaptively secure SCC scheme for polynomial differentiation (based on Lemma 4). For completeness, we also present a selectively secure scheme for polynomial differentiation in the full version of the paper [28].

$\operatorname{Algorithm}(\mathrm{PK}, \mathrm{SK}) \leftarrow \operatorname{KeyGen}(\lambda, \mathcal{F})$ : Same as in Section 3

Algorithm $\mathrm{FK}(f) \leftarrow \operatorname{Setup}(\mathrm{SK}, \mathrm{PK}, f)$ : Same as in Section 3 .

$\operatorname{Algorithm}(v, w) \leftarrow \operatorname{Compute}(\mathrm{PK}, f, \mathbf{a}, k$, ind $):$ In addition to the point $\mathbf{a} \in \mathbb{Z}_{p}^{n}$, the Compute algorithm here takes in two additional parameters $k$ and ind, indicating the evaluation of the $k$-th derivative of the polynomial with respect to variable $x_{\text {ind }}$ at $\mathbf{a}$. 
Without loss of generality, below we assume ind $=n$. In other words, the algorithm should evaluate the $k$-th partial derivative with respect to $x_{n}$ at point a. First, the algorithm computes randomness $r_{i}$ as

$$
r_{i}=\mathrm{H}(\mathbf{a} \| \text { ind }\|k\| i) \quad \forall 1 \leq i \leq n-2,
$$

where $\mathbf{H}:\{0,1\}^{*} \rightarrow \mathbb{Z}_{p}$ is a hash function (later modeled as a random oracle). Due to Lemma 4, $f(\mathbf{x})$ can be expressed as $f(\mathbf{x})=\sum_{i=1}^{n-2}\left[r_{i}\left(x_{i}-a_{i}\right)+x_{i+1}-a_{i+1}\right] u_{i}(\mathbf{x})+$ $\left(x_{n-1}-a_{n-1}\right) u_{n-1}(\mathbf{x})+\left(x_{n}-a_{n}\right)^{k+1} q\left(x_{n}\right)+\sum_{i=0}^{k} c_{i} x_{n}^{i}$. The signature $w$ for correct derivative computation is the following tuple:

$$
w=\left(g^{u_{1}(\mathbf{t})}, \ldots, g^{u_{n-2}(\mathbf{t})}, g^{q\left(t_{n}\right)}, c_{k-1}, \ldots, c_{1}, c_{0}, \text { polynomial } u_{n-1}(\mathbf{x})\right),
$$

where polynomial $u_{n-1}(\mathbf{x})$ is a description of the polynomial containing the corresponding coefficients. Note that by Lemma 4 polynomial $u_{n-1}(\mathbf{x})$ contains up to $d^{2}$ terms. Also, the signature does not contain the term $c_{k}$ - this can be implicitly retrieved by the result $v$ since $c_{k}=v / k$ !. Finally, the result of the computation $v$ is returned.

Algorithm Verify $(\mathrm{PK}, \mathrm{FK}(f), \mathbf{a}, k$, ind, $v, w)$ : Let $c_{k}=\frac{v}{k !}$. To verify that $v$ is indeed the outcome of the $k$-th partial derivative on variable $x_{\text {ind }}$ (ind $=n$ ) evaluated at point $\mathbf{a} \in \mathbb{Z}_{p}^{n}$, perform the following steps.

Parse $w$ as $\left(w_{1}, \ldots, w_{n-2}, w_{n}, c_{k-1}, \ldots, c_{1}, c_{0}\right.$, polynomial $\left.u_{n-1}(\mathbf{x})\right)$.

Compute the $r_{i}$ values in the same way as in Equation 5.8, i.e., $r_{i}=\mathrm{H}(\mathbf{a} \|$ ind $\|k\| i)$ for $1 \leq i \leq n-2$.

Check if e $(\mathrm{FK}(f), g)$ equals the following quantity (where $\left.\mathrm{L}=\prod_{i=0}^{k} \mathrm{e}\left(g^{t_{n}^{i}}, g\right)^{c_{i}}\right)$ :

$$
\prod_{i=1}^{n-2} \mathrm{e}\left(g^{r_{i}\left(t_{i}-a_{i}\right)+t_{i+1}-a_{i+1}}, w_{i}\right) \cdot \mathrm{e}\left(g^{t_{n-1}-a_{n-1}}, g^{u_{n-1}(\mathbf{t})}\right) \cdot \mathrm{e}\left(g^{\left(t_{n}-a_{n}\right)^{k+1}}, w_{n}\right) \cdot \mathrm{L}
$$

The above quantity can be easily computed with the public keys in $O\left(n+d^{2}\right)$ time, since $u_{n-1}(\mathbf{x})$ is a polynomial containing $d^{2}$ terms and $k \leq d$ (see Section 6). The algorithm accepts $v$ and outputs 1 if the above equation holds; otherwise, it rejects.

Algorithm $\mathrm{FK}\left(f^{\prime}\right) \leftarrow$ Update $\left(\mathrm{SK}, \mathrm{PK}, \mathrm{FK}(f), f^{\prime}\right)$ : Same as in Section 3 .

Theorem 4. There exists an SCC scheme for the differentiation of multivariate polynomials such that (1) It is correct according to Definition 2. (2) It is adaptively secure according to Definition 3 under the $\ell-S B D H$ assumption and in the random oracle model.

Corollary 1. There exists a PVC scheme for the differentiation of multivariate polynomials such that (1) It is correct according to Definition 8. (2) It is adaptively secure according to Definition 9 and under the $\ell-S B D H$ assumption.

\section{Asymptotic Cost Analysis}

In this section, we analyze the asymptotic cost of our schemes. Clearly, the worst-case complexity of KeyGen is $O\left(\left(\begin{array}{c}n+d \\ d\end{array}\right)\right)$, since the set $\mathcal{W}_{n, d}$ should contain one term for 
every possible term of the polynomial in $n$ variables and total degree $d$. Similarly algorithm Setup takes $O\left(\left(\begin{array}{c}n+d \\ d\end{array}\right)\right)$ time to execute in the worst case. In practice, both these complexities can be $O(m)$, where $m$ is the number of the terms contained in the polynomial - see the full version of the paper [28] for minimizing the size of $\mathcal{W}_{n, d}$.

Also for our adaptive security schemes, the size of the signature is $O(n)$, and the client performs $O(n)$ amount of work to verify it using algorithm Verify (these costs are $O(n+d)$ for derivative computation). For our adaptive security schemes, the size of the signature increases to $O(n+d)$, and the client performs $O(n+d)$ amount of work to verify it (again, these costs $O\left(n+d^{2}\right)$ for derivative computation).

As for algorithm Compute, it needs to decompose the polynomial according to Lemmata 1, 2, 3, 4 (depending on which scheme we are using). This polynomial decomposition dominates the asymptotic performance. To perform the polynomial decomposition, the server performs $n$ polynomial divisions. If we use the naive polynomial division algorithm, since each variable can have degree up to $d$, each polynomial division involves $d$ steps, and each step takes time proportional to the number of terms in the polynomial, namely, $O\left(\left(\begin{array}{c}n+d \\ d\end{array}\right)\right)$. Therefore, the polynomial decomposition (Lemma 1) can be achieved in $O\left(n d\left(\begin{array}{c}n+d \\ d\end{array}\right)\right)$ time using the naive algorithm. However, in cases where $d>\log n$, one can use the FFT method to perform polynomial division, resulting in $O\left(n \log n\left(\begin{array}{c}n+d \\ d\end{array}\right)\right)$ computation time. Finally, algorithm Update takes constant time to update a constant number of coefficients.

\section{Extensions and Observations}

\subsection{I/O Privacy}

In our constructions, the client's sensitive input is in plaintext, directly readable by the untrusted server. To offer input and output privacy, we could potentially use a fully-homomorphic public-key encryption scheme [16] (FHE scheme) so that algorithm Compute executed by the untrusted server could operate on encrypted points. In this way, everybody that knows pk could send queries to the server. After Compute executes on the encryption of some point $\overline{\mathbf{a}}$, it outputs the encrypted signature $w$ of the value $\bar{v}=f(\overline{\mathbf{a}})$ under the public key pk, allowing only the owner of the secret key to decrypt and retrieve (and verify) the output of the computation. This could have various applications which we highlight in the Appendix of the full version of the paper [28].

\subsection{Removing the Random Oracles Through Stronger Assumptions}

We now observe that if we are willing to (i) use subexponential assumptions and (ii) restrict the size of the domain of the inputs of our polynomials to be subexponential (now it is exponential), we can remove the random oracle from our adaptively secure constructions. The subexponential assumption we use can be stated as follows:

Definition 5 ( $\delta$-subexponential bilinear $\ell$-strong Diffie-Hellman assumption). Suppose $k$ is the security parameter, let $0<\delta<\frac{\log k-1}{\log k}$ and let $\left(p, \mathbb{G}, \mathbb{G}_{T}, \mathrm{e}, g\right)$ be a uniformly randomly generated tuple of bilinear pairings parameters. Given the elements $g, g^{t}, \ldots, g^{t^{\ell}} \in \mathbb{G}$ for some $t$ chosen at random from $\mathbb{Z}_{p}^{*}$, for $\ell=\operatorname{poly}(k)$, there is no 
algorithm running in time less than $2^{2 k^{\delta}}$ that can output the pair $\left(c, \mathrm{e}(g, g)^{1 /(t+c)}\right) \in$ $\mathbb{Z}_{p}^{*} \backslash\{-t\} \times \mathbb{G}_{T}$, except with negligible probability neg $(k)$.

Note that in the above definition, we require $\delta<\frac{\log k-1}{\log k}$ so that $2 k^{\delta}<k$.

Theorem 5 (Adaptive security in the standard model). Let $\mathbf{x}$ be the input to our polynomial. For $\mathbf{x}$ belonging to a domain of subexponential size, our selectively secure scheme (Section 3) is adaptively secure in the standard model and assuming the $\delta$-subexponential bilinear $\ell$-strong Diffie-Hellman assumption. Namely, for all PPT adversaries, we can build a simulator running in subexponential time that breaks the $\delta$-subexponential bilinear $\ell$-strong Diffie-Hellman assumption (see Definition 5).

Proof. Suppose we have $n$ variables $x_{1}, x_{2}, \ldots, x_{n}$, and each one of which can take values in $[0,1, \ldots, m-1]$. Assume that $m^{n}=2^{k^{\delta}}$, yielding $n \log m=k^{\delta}$. To build the desired simulator, we modify the initialization phase of our selective security proof in Section 3.3. We do not require the adversary to commit to an initial point a. Instead the simulator guesses the point a that the adversary is going to output later as a forgery - and the simulator aborts if the guess is wrong. Clearly, the guess is successful with probability $2^{-k^{\delta}}$. Therefore the simulation, in expectation, takes $2^{k^{\delta}}$ time to succeed. Since the adversary runs in at most polynomial time (see our adaptive security definition), it follows that we have derived an algorithm that runs in poly $(k) 2^{k^{\delta}}$ time and breaks the assumption. Note that this is a contradiction since the function poly $(k) 2^{k^{\delta}}=o\left(2^{2 k^{\delta}}\right)$. This completes our proof.

The same technique was also described by Boneh and Boyen [7] to achieve adaptive security in their IBE scheme.

Acknowledgments. This work was supported by Intel through the ISTC for Secure Computing, by the National Science Foundation under grants CCF-0424422, 0842695, 0808617 and CNS-1228485, by the Air Force Office of Scientific Research (AFOSR) under MURI award FA9550-09-1-0539, by the MURI program under AFOSR grant FA9550-08-1-0352, by the Center for Geometric Computing at Brown University, and by a NetApp Faculty Fellowship. The authors thank Xavier Boyen, Basilis Gidas, Dawn Song and Nikos Triandopoulos for useful discussions, and the TCC 2013 reviewers for their feedback. Any opinions, findings, and conclusions or recommendations expressed in this material are those of the authors and do not necessarily reflect the views of the sponsors.

\section{References}

1. Applebaum, B., Ishai, Y., Kushilevitz, E.: From Secrecy to Soundness: Efficient Verification via Secure Computation. In: Abramsky, S., Gavoille, C., Kirchner, C., Meyer auf der Heide, F., Spirakis, P.G. (eds.) ICALP 2010. LNCS, vol. 6198, pp. 152-163. Springer, Heidelberg (2010)

2. Atallah, M.J., Cho, Y., Kundu, A.: Efficient data authentication in an environment of untrusted third-party distributors. In: ICDE, pp. 696-704 (2008) 
3. Benabbas, S., Gennaro, R., Vahlis, Y.: Verifiable Delegation of Computation over Large Datasets. In: Rogaway, P. (ed.) CRYPTO 2011. LNCS, vol. 6841, pp. 111-131. Springer, Heidelberg (2011)

4. Bitansky, N., Canetti, R., Chiesa, A., Tromer, E.: From extractable collision resistance to succinct non-interactive arguments of knowledge, and back again. In: ITCS, pp. 326-349 (2012)

5. Bitansky, N., Canetti, R., Chiesa, A., Tromer, E.: Recursive composition and bootstrapping for snarks and proof-carrying data. IACR Cryptology ePrint Archive 2012:95 (2012)

6. Boneh, D., Boyen, X.: Short signatures without random oracles and the SDH assumption in bilinear groups. J. Cryptology 21(2), 149-177 (2008)

7. Boneh, D., Boyen, X.: Efficient selective identity-based encryption without random oracles. J. Cryptology 24(4), 659-693 (2011)

8. Boneh, D., Waters, B.: Conjunctive, Subset, and Range Queries on Encrypted Data. In: Vadhan, S.P. (ed.) TCC 2007. LNCS, vol. 4392, pp. 535-554. Springer, Heidelberg (2007)

9. Canetti, R., Riva, B., Rothblum, G.N.: Two 1-round protocols for delegation of computation. IACR Cryptology ePrint Archive 2011:518 (2011)

10. Chung, K.-M., Kalai, Y., Vadhan, S.: Improved Delegation of Computation Using Fully Homomorphic Encryption. In: Rabin, T. (ed.) CRYPTO 2010. LNCS, vol. 6223, pp. 483-501. Springer, Heidelberg (2010)

11. Damgård, I.B.: Towards Practical Public Key Systems Secure against Chosen Ciphertext Attacks. In: Feigenbaum, J. (ed.) CRYPTO 1991. LNCS, vol. 576, pp. 445-456. Springer, Heidelberg (1992)

12. Fiore, D., Gennaro, R.: Improved publicly verifiable delegation of large polynomials and matrix computations. IACR Cryptology ePrint Archive 2012:434 (2012)

13. Fiore, D., Gennaro, R.: Publicly verifiable delegation of large polynomials and matrix computations, with applications. In: CCS, pp. 501-512 (2012)

14. Gennaro, R., Gentry, C., Parno, B.: Non-interactive Verifiable Computing: Outsourcing Computation to Untrusted Workers. In: Rabin, T. (ed.) CRYPTO 2010. LNCS, vol. 6223, pp. 465-482. Springer, Heidelberg (2010)

15. Gennaro, R., Gentry, C., Parno, B., Raykova, M.: Quadratic span programs and succinct NIZKs without PCPs. IACR Cryptology ePrint Archive 2012:215 (2012)

16. Gentry, C.: Fully homomorphic encryption using ideal lattices. In: STOC, pp. 169-178 (2009)

17. Gentry, C., Wichs, D.: Separating succinct non-interactive arguments from all falsifiable assumptions. In: STOC, pp. 99-108 (2011)

18. Goodrich, M.T., Tamassia, R., Schwerin, A.: Implementation of an authenticated dictionary with skip lists and commutative hashing. In: DISCEX II, pp. 68-82 (2001)

19. Goodrich, M.T., Tamassia, R., Triandopoulos, N.: Super-Efficient Verification of Dynamic Outsourced Databases. In: Malkin, T. (ed.) CT-RSA 2008. LNCS, vol. 4964, pp. 407-424. Springer, Heidelberg (2008)

20. Goodrich, M.T., Tamassia, R., Triandopoulos, N.: Efficient authenticated data structures for graph connectivity and geometric search problems. Algorithmica 60(3), 505-552 (2011)

21. Goyal, V., Jain, A., Pandey, O., Sahai, A.: Bounded Ciphertext Policy Attribute Based Encryption. In: Aceto, L., Damgård, I., Goldberg, L.A., Halldórsson, M.M., Ingólfsdóttir, A., Walukiewicz, I. (eds.) ICALP 2008, Part II. LNCS, vol. 5126, pp. 579-591. Springer, Heidelberg (2008)

22. Groth, J.: Short Non-interactive Zero-Knowledge Proofs. In: Abe, M. (ed.) ASIACRYPT 2010. LNCS, vol. 6477, pp. 341-358. Springer, Heidelberg (2010)

23. Kate, A., Zaverucha, G.M., Goldberg, I.: Constant-Size Commitments to Polynomials and Their Applications. In: Abe, M. (ed.) ASIACRYPT 2010. LNCS, vol. 6477, pp. 177-194. Springer, Heidelberg (2010) 
24. Lewko, A., Waters, B.: New Proof Methods for Attribute-Based Encryption: Achieving Full Security through Selective Techniques. In: Safavi-Naini, R. (ed.) CRYPTO 2012. LNCS, vol. 7417, pp. 180-198. Springer, Heidelberg (2012)

25. Yiu, M.L., Lin, Y., Mouratidis, K.: Efficient verification of shortest path search via authenticated hints. In: ICDE, pp. 237-248 (2010)

26. Merkle, R.C.: A Certified Digital Signature. In: Brassard, G. (ed.) CRYPTO 1989. LNCS, vol. 435, pp. 218-238. Springer, Heidelberg (1990)

27. Micali, S.: Computationally sound proofs. SIAM J. Comput. 30(4), 1253-1298 (2000)

28. Papamanthou, C., Shi, E., Tamassia, R.: Signatures of correct computation. IACR Cryptology ePrint Archive 2011: 587 (2011)

29. Papamanthou, C., Tamassia, R., Triandopoulos, N.: Authenticated hash tables. In: CCS, pp. 437-448 (2008)

30. Papamanthou, C., Tamassia, R., Triandopoulos, N.: Optimal Verification of Operations on Dynamic Sets. In: Rogaway, P. (ed.) CRYPTO 2011. LNCS, vol. 6841, pp. 91-110. Springer, Heidelberg (2011)

31. Parno, B., Raykova, M., Vaikuntanathan, V.: How to Delegate and Verify in Public: Verifiable Computation from Attribute-Based Encryption. In: Cramer, R. (ed.) TCC 2012. LNCS, vol. 7194, pp. 422-439. Springer, Heidelberg (2012)

32. Sahai, A., Waters, B.: Fuzzy Identity-Based Encryption. In: Cramer, R. (ed.) EUROCRYPT 2005. LNCS, vol. 3494, pp. 457-473. Springer, Heidelberg (2005)

33. Tamassia, R.: Authenticated Data Structures. In: Di Battista, G., Zwick, U. (eds.) ESA 2003. LNCS, vol. 2832, pp. 2-5. Springer, Heidelberg (2003)

34. Tamassia, R., Triandopoulos, N.: Efficient Content Authentication in Peer-to-Peer Networks. In: Katz, J., Yung, M. (eds.) ACNS 2007. LNCS, vol. 4521, pp. 354-372. Springer, Heidelberg (2007)

35. Tamassia, R., Triandopoulos, N.: Certification and authentication of data structures. In: Proc. Alberto Mendelzon Workshop on Foundations of Data Management (2010)

\section{Appendix}

Definition 6 (Selective security of an SCC scheme). Let $\lambda$ be the security parameter and let $\mathcal{P}$ be an SCC scheme (KeyGen, Setup, Compute, Verify, Update) for a function family $\mathcal{F}$. We say that $\mathcal{P}$ is selectively-secure if no PPT adversary $\mathcal{A}$ has more than negligible probability neg $(\lambda)$ in winning the following game between $\mathcal{A}$ and a challenger:

1. Initialization. The adversary $\mathcal{A}$ commits to a point $\mathbf{b}$. The challenger runs algorithm KeyGen which outputs (PK, SK) and gives $\mathrm{PK}$ to $\mathcal{A}$ but maintains SK secret;

2. Setup and Update. The adversary $\mathcal{A}$ initially makes an oracle query to algorithm $\operatorname{Setup}\left(\mathrm{SK}, \mathrm{PK}, f_{0}\right)$, specifying an initial function $f_{0} \in \mathcal{F}$, outputting $\mathrm{FK}\left(f_{0}\right)$. Then, for $i=1, \ldots, k$, where $k=$ poly $(\lambda)$, he makes a polynomial number of oracle queries to the Update $\left(\mathrm{SK}, \mathrm{PK}, \mathrm{FK}\left(f_{i-1}\right), f_{i}\right)$ algorithm, each time specifying $f_{i} \in$ $\mathcal{F}$. The challenger answers the queries by returning the resulting $\operatorname{FK}\left(f_{i}\right)$;

3. Forgery. The adversary $\mathcal{A}$ outputs a forgery $(\mathbf{b}, v, w)$ for point $\mathbf{b}$ that he committed in the initialization phase, for some function $f_{i}$ previously queried where $0 \leq i \leq k$.

The adversary $\mathcal{A}$ wins if $1 \leftarrow \operatorname{Verify}\left(\mathrm{PK}, \mathrm{FK}\left(f_{i}\right), \mathbf{b}, v, w\right)$ and $f_{i}(\mathbf{b}) \neq v$.

Definition 7 (PVC scheme). We define a PVC scheme for a function family $\mathcal{F}$ to be a tuple of six PPT algorithms (KeyGen, Setup, Challenge, Compute, Verify, Update) with the following specification: 
1. (PK, SK $) \leftarrow \operatorname{KeyGen}(\lambda, \mathcal{F}):$ Algorithm KeyGen takes as input the security parameter $\lambda$ and a function family $\mathcal{F}$. It outputs a public/secret key pair (PK, SK). KeyGen is run only once at system initialization by a trusted source;

2. $\mathrm{FK}(f) \leftarrow \operatorname{Setup}(\mathrm{SK}, \mathrm{PK}, f):$ Algorithm Setup (run by a trusted source) takes as input the secret key SK, the public key $\mathrm{PK}$, and a function $f \in \mathcal{F}$. It outputs the function public key $\mathrm{FK}(f)$ for the function $f$;

3. $\operatorname{chal}(\mathbf{a}) \leftarrow$ Challenge $(\mathrm{PK}, \mathbf{a}):$ Algorithm Challenge (run by the verifier) takes as input a value $\mathbf{a} \in \operatorname{domain}(f)$. It outputs a challenge chal $(\mathbf{a})$ corresponding to $\mathbf{a}$;

4. $(v, w) \leftarrow$ Compute $(\mathrm{PK}, f, \mathbf{a}, \operatorname{chal}(\mathbf{a}))$ : Algorithm Compute (run by an untrusted server) takes as input the public key PK, a function $f \in \mathcal{F}$ and a value a $\in$ domain $(f)$. It outputs a pair $(v, w)$, where $v=f(\mathbf{a})$ and $w$ is a signature;

5. $\{0,1\} \leftarrow \operatorname{Verify}(\mathrm{PK}, \mathrm{FK}(f)$, a, chal $(\mathbf{a}), v, w)$ : Algorithm Verify (run by the verifier) takes as input the public key $\mathrm{PK}$, function public key $\mathrm{FK}(f)$, value $\mathbf{a} \in \operatorname{domain}(f)$, a claimed result $v$ and a signature $w$. It outputs 0 or 1 ;

6. $\mathrm{FK}\left(f^{\prime}\right) \leftarrow$ Update $\left(\mathrm{SK}, \mathrm{PK}, \mathrm{FK}(f), f^{\prime}\right):$ Algorithm Update (run by the trusted source) takes as input the secret key SK, the public key $\mathrm{PK}$, the function public key $\mathrm{FK}(f)$ for the old function $f$ and the updated function description $f^{\prime}$. It outputs the updated function public key $\mathrm{FK}\left(f^{\prime}\right)$.

Definition 8 (Correctness of a PVC scheme). Let $\lambda$ be the security parameter and let $\mathcal{P}$ be a PVC scheme (KeyGen, Setup, Challenge, Compute, Verify, Update) for a function family $\mathcal{F}$. Let $(\mathrm{PK}, \mathrm{SK}) \leftarrow \operatorname{KeyGen}(\lambda, \mathcal{F})$. For all $i=1, \ldots$, poly $(\lambda)$, for any function $f_{i} \in \mathcal{F}$, suppose $\mathrm{FK}\left(f_{i}\right)$ is the output of Update(SK, PK, $\left.\mathrm{FK}\left(f_{i-1}\right), f_{i}\right)$, where $\mathrm{FK}\left(f_{0}\right)$ is output by algorithm $\operatorname{Setup}\left(\mathrm{SK}, \mathrm{PK}, f_{0}\right)$ for some $f_{0} \in \mathcal{F}$. We say that $\mathcal{P}$ is correct, if for any $i=0, \ldots$, poly $(\lambda)$, for any $\mathbf{a} \in \operatorname{domain}\left(f_{i}\right)$, for any chal $(\mathbf{a})$ output by Challenge $(\mathrm{PK}, \mathbf{a})$, it is $1 \leftarrow \operatorname{Verify}\left(\mathrm{PK}, \mathrm{FK}\left(f_{i}\right)\right.$, a, chal $\left.\left.(\mathbf{a})\right), v, w\right)$, where $\left.(v, w) \leftarrow \operatorname{Compute}\left(\mathrm{PK}, f_{i}, \mathbf{a}, \operatorname{chal}(\mathbf{a})\right)\right)$.

Definition 9 (Adaptive security of a PVC scheme). Let $\lambda$ be the security parameter and let $\mathcal{P}$ be a PVC scheme (KeyGen, Setup, Challenge, Compute, Verify, Update) for a function family $\mathcal{F}$. We say that $\mathcal{P}$ is adaptively secure if no PPT adversary $\mathcal{A}$ has more than negligible probability neg $(\lambda)$ in winning the following security game, played between the adversary $\mathcal{A}$ and a challenger:

1. Initialization. The challenger runs algorithm KeyGen which outputs (PK, SK) and then gives $\mathrm{PK}$ to the adversary but maintains SK secret;

2. Setup and Update. The adversary initially makes an oracle query to algorithm $\operatorname{Setup}\left(\mathrm{SK}, \mathrm{PK}, f_{0}\right)$, specifying an initial function $f_{0} \in \mathcal{F}$, outputting $\mathrm{FK}\left(f_{0}\right)$. Then, for $i=1, \ldots, k$, where $k=$ poly $(\lambda)$, he makes a polynomial number of oracle queries to the Update $\left(\mathrm{SK}, \mathrm{PK}, \mathrm{FK}\left(f_{i-1}\right), f_{i}\right)$ algorithm, each time specifying $f_{i} \in$ $\mathcal{F}$. The challenger answers the queries by returning the resulting $\mathrm{FK}\left(f_{i}\right)$;

3. Challenge and Forgery. The adversary $\mathcal{A}$ outputs a point $\mathbf{b}$ and sends it to the challenger. The challenger returns $\operatorname{chal}(\mathbf{b})$ output by Challenge. The adversary $\mathcal{A}$ outputs the forgery $(\mathbf{b}, \operatorname{chal}(\mathbf{b}), v, w)$ for one of the functions $f_{i}(0 \leq i \leq k)$ that has been queried.

The adversary $\mathcal{A}$ wins if $1 \leftarrow \operatorname{Verify}\left(\mathrm{PK}, \mathrm{FK}\left(f_{i}\right)\right.$, $\mathbf{b}$, chal $\left.(\mathbf{b}), v, w\right)$ and $f_{i}(\mathbf{b}) \neq v$. 